\section{Don't forget the Universe}

SIR - In June of this year the scientific programme committee of the European Space Agency (ESA) approved the COBRAS-SAMBA satellite as the agency's third medium-size mission, and the first devoted to the critical problems of cosmology. COBRAS/SAMBA will map the whole sky with unprecedented precision over the wavelength range from 3 to 0.1 millimetres. After the extraordinary success of NASA's satellite COBE, we now know that the design specification of COBRAS/SAMBA should allow all the observationally accessible structure of the Universe at an age of only 300,000 years to be mapped in detail. Such maps are expected to yield precise measures of the age, matter content and curvature of our Universe, as well as determining whether a cosmological constant in Einstein's equations has affected cosmic evolution. They will demonstrate whether our current understanding of the history of the Universe is correct. Such a demonstration would clearly have profound philosophical implications going well beyond technical cosmology and comparable, perhaps, to Magellan's demonstration that the Earth is round. Such a crucial addition to our knowledge of the world can occur only once, and COBRAS/SAMBA has the chance to make this step.

Unfortunately, the Ariane 5 disaster, occurring just before the ESA committee meetings, has caused serious planning problems. The scientific programme committee, while approving COBRAS/SAMBA, decided that final confirmation must await assessment of the effects of the loss of Cluster, a mission to study the structure of the Earth's magnetosphere which was destroyed with Ariane 5. Your 14 November issue reported that ESA's external space science advisory committee recommended that a replacement for Cluster be launched in 1999, and that the cost of ECU210 million be found by delaying the Far Infrared Space Telescope by six months and the fourth medium mission (expected to be a planetary mission) by two years (Nature 384, 99). We are writing to emphasize that the costs of the Cluster replacement should not delay the whole of ESA's long-range Horizon 2000 plan. In particular it is critical that COBRAS/SAMBA be launched at or before the current outline date of 2004. (It was to be 2003 before a cost-saving exercise carried out by ESA earlier this year).

COBE generated worldwide interest in using the microwave background to understand the origin of our Universe. Cosmology has now become highly competitive and is supported strongly in many countries. There are, worldwide, rapid developments in microwave technology, and in experiments on the ground, in balloons, and in simpler satellites. Any delay could jeopardize COBRAS/SAMBA's status as the definitive mission; a unique opportunity for European science would then have been lost.

\section{Martin Rees}

Institute of Astronomy,

University of Cambridge,

Madingley Road,

Cambridge $\mathrm{CB} 3 \mathrm{OHA}$, UK

Rashid Sunyaev

\section{Simon White}

Max-Planck-Institut für Astrophysik,

Karl-Schwarzchild-Strasse 1,

85470 Garching bei München,

Germany

\section{First for biotech}

SIR - I was pleased to learn that the British government is to appoint a Human Genetics Commission to regulate the complex field of genetic diagnosis and technologies, in addition to the Advisory Committee on Genetic Testing (see Nature 381, 640; 1996). Although you stated that the latter committee was thought to be the "first of its kind", with responsibility for ensuring that genetic screening tests are "supplied safely and used ethically" (Nature 379, 195; 1996), for more than a year the Norwegian National Board of Health has had a Medical Biotechnology Advisory Board with these tasks as well as broader responsibilities like those of the Human Genetics Commission.

The Norwegian regulations took effect on 1 September 1994, and cover human reproductive technology, research on embryos, all kinds of genetic testing on embryos, fetuses and born people, as well as gene therapy. Although it is important that regulations must not make valuable clinical practice too difficult, it seems clear that this field should not be left open to market forces. We are convinced that such a law is necessary, and that regulation will prevent many of the difficulties reported from other countries (see Nature 378, 120; 1995).

The borderline between genetic variation and disease cannot be conveniently defined in legal terms, and in decisions on how to regulate this field there is no substitute for the detailed clinical and scientific knowledge provided by a good advisory board. Summing up our first year's experience, the advisory group has been indispensable, and we are convinced that the British authorities will not regret the decision to appoint their group.

\section{Ola Myklebost}

\section{(Secretary)}

Medical Biotechnology Advisory Board, Norwegian Board of Health, PO Box 8128 Dep, N-0032 Oslo, Norway e-mail: olam@radium.uio.no

\section{Police yourselves}

SIR - It was not my intention in my report on "Fringe science in the 104th Congress", discussed in your leading article "Don't ban sceptics" (Nature 383, 745; 1996), to imply that dissenting scientific viewpoints should be suppressed. Nor was it my intention to reflect adversely on the character, integrity, credibility or qualifications of the "sceptic" scientists who testified before our committee. My point was rather that scientific disagreements are better resolved within the scientific community than by Congress.

Members of Congress are not particularly well qualified to make scientific judgements or to conduct peer review of scientific assessments. Our best assurance of objective, highquality science remains the peer-review system, as imperfect as it may be. In that regard, I encourage those who doubt the socalled 'scientific consensus' to spend less time spinning their views to the media and more time convincing scientific colleagues of the merit of those views through time-tested scientific methods such as proposing and testing alternative hypotheses.

To be sure, Congress does have a role in ensuring that the scientific process is truly open to legitimate alternative viewpoints. The report also points out that scientific certainty is one among many legitimate policy issues that Congress must consider in making regulatory policies. In that light, Congress should always be open to hearing a variety of policy perspectives of those with experience and expertise in the science and policy arena.

\section{George E. Brown Jr}

US House of Representatives,

Committee on Science,

Suite 2320, Rayburn House Office Bldg,

Washington DC 20515-6301, USA

\section{Next step in space}

SIR - Your item on the space science workshop being hosted by the National Research Council (NRC) in advance of the forthcoming 'space summit' may convey a misleading impression (Nature 383, 372; 1996).

The expert group convened at the request of the National Aeronautics and Space Administration (NASA) will carry out a technical assessment of current NASA programmes in light of previous NRC recommendations, and prepare a discussion paper outlining "appropriate next steps" in broad terms. The group is not charged with setting mission or programme priorities or developing a budget analysis or recommendations. The paper will not be a formal NRC report but a summary of the workshop proceedings.

Marc S. Allen

National Research Council, 2101 Constitution Avenue, Washington DC 20418, USA 